\title{
Effect of Catalytic Layer Thickness on Diameter of Vertically Aligned Individual Carbon Nanotubes
}

\author{
Hyun Kyung Jung ${ }^{1}$ and Hyung Woo Lee ${ }^{2,3}$ \\ ${ }^{1}$ Department of Diagnostic Radiology, College of Medicine, Haeundae Paik Hospital, Inje University, Busan 612-030, Republic of Korea \\ ${ }^{2}$ Department of Nanomaterials Engineering, College of Nanoscience and Nanotechnology, Pusan National University, \\ Busan 609-73, Republic of Korea \\ ${ }^{3}$ Department of Nanofusion Technology, College of Nanoscience and Nanotechnology, Pusan National University, \\ Busan 609-735, Republic of Korea \\ Correspondence should be addressed to Hyung Woo Lee; lhw2010@pusan.ac.kr
}

Received 11 December 2013; Accepted 7 February 2014; Published 10 March 2014

Academic Editor: Chunyi Zhi

Copyright ( 2014 H. K. Jung and H. W. Lee. This is an open access article distributed under the Creative Commons Attribution License, which permits unrestricted use, distribution, and reproduction in any medium, provided the original work is properly cited.

\begin{abstract}
The effect of catalytic thin film thickness on the diameter control of individual carbon nanotubes grown by plasma enhanced chemical vapor deposition was investigated. Individual carbon nanotubes were grown on catalytic nanodot arrays, which were fabricated by e-beam lithography and e-beam evaporation. During e-beam evaporation of the nanodot pattern, more catalytic metal was deposited at the edge of the nanodots than the desired catalyst thickness. Because of this phenomenon, carbon atoms diffused faster near the center of the dots than at the edge of the dots. The carbon atoms, which were gathered at the interface between the catalytic nanodot and the diffusion barrier, accumulated near the center of the dot and lifted the catalyst off. From the experiments, an individual carbon nanotube with the same diameter as that of the catalytic nanodot was obtained from a $5 \mathrm{~nm}$ thick catalytic nanodot; however, an individual carbon nanotube with a smaller diameter ( $40 \%$ reduction) was obtained from a $50 \mathrm{~nm}$ thick nanodot. We found that the thicker the catalytic layer, the greater the reduction in diameter of the carbon nanotubes. The diameter-controlled carbon nanotubes could have applications in bio- and nanomaterial scanning and as a contrast medium for magnetic resonance imaging.
\end{abstract}

\section{Introduction}

Recently, various nanomaterials have been widely used in nanoscience, engineering, and technology. In particular, carbon nanotubes (CNTs) are the most popular material in the nanotechnology field. Since discovered in the soot of an arc discharge apparatus [1], CNTs have been used in many nanotechnology applications such as transistors and chemical or biosensors, and as atomic force microscopy tips because of their remarkable electrical, mechanical, chemical, and thermal properties [2-6]. In many cases, however, it is difficult to control the nanotubes' locations and orientations. Recently, an attempt to manipulate nanotubes has been made by polymer encapsulation [7].

In the last decade, chemical vapor deposition (CVD) techniques, particularly plasma enhanced chemical vapor deposition (PECVD), have been widely used to grow CNTs
[8]. The controlled synthesis of CNTs is an essential step for realizing CNT-based devices and technologies. In particular, vertically aligned carbon nanotube growth is important for applications such as field emission displays (FEDs) and scanning probe microscopy (SPM) [9]. However, because of the complicated coupled parameters that are required to grow CNTs $[10,11]$, it is not easy to control the synthesis of CNTs. Moreover, vertically aligned individual carbon nanotubes are not easily grown because of the lack of knowledge about the mechanism of interaction between the thin catalytic layer and individual dots. Several papers have shown that the growth depends on parameters such as substrate temperature, gas ratio of hydrocarbon gas and dilution gas, pretreatment using plasma, and catalyst film thickness [12-17]. For growing individual carbon nanotubes, a single nanodot is needed to control the CNT's diameter and to make a catalytic island without undergoing etching $[18,19]$. Although patterned dots 
are used in CNT growth, CNTs grown by PECVD still have various diameters depending on the growth conditions.

In this paper, we report the effect of the catalytic layer thickness on the diameter of individual carbon nanotubes grown using direct current PECVD (DC-PECVD). Specifically, we show that the thickness of the catalytic nanodots affects the diameter of the resulting vertically aligned individual carbon nanotubes. From the field emission scanning electron microscopy (FESEM) and atomic force microscopy (AFM) images, we verified the relation between the carbon nanotube diameter and the catalytic layer thickness of nanodots.

\section{Experimental Section}

2.1. Fabrication Processes for Catalytic Nanodots. Co, Fe, Mo, and $\mathrm{Ni}$ are generally used as catalysts for carbon nanotube growth. In this study, we used $\mathrm{Ni}$ as the catalytic metal. For growing CNTs at determined locations, we patterned nanodots with diameters of $400 \mathrm{~nm}$ on a p-type, borondoped silicon (100) wafer. Titanium nitride (TiN) with 200 $\mathrm{nm}$ thickness was deposited before catalytic layer deposition to provide a buffer layer on the $\mathrm{SiO}_{2} / \mathrm{Si}$ substrate in order to prevent catalyst silicide formation under high growth temperature conditions. To make small nanodots, electron beam lithography (Raith 150) was used on an e-beam resist (3\% 950,000-MW (molecular weight) PMMA (polymethyl methacrylate) in chlorobenzene) deposited on the titanium nitride/silicon dioxide/silicon wafer. Through a series of ebeam exposures, we found that an e-beam area dose of $150 \mu \mathrm{As} / \mathrm{cm}^{2}$ was needed for making dots with $400 \mathrm{~nm}$ diameters. Next, the patterns were developed for 80 seconds in a mixed solution of methyl isobutyl ketone (MIBK) and isopropyl alcohol (IPA) with a ratio of $1: 3$. Subsequently, $\mathrm{Ni}$ with 5 50 nm thickness was deposited by electron beam evaporation. Finally, Ni nanodots were produced after metal lift off in acetone or $N$-methylpyrrolidone (NMP). Figure 1 shows a schematic diagram of the microfabrication processes for nanodot arrays and subsequent carbon nanotube growth by PECVD.

\subsection{Vertically Aligned Carbon Nanotube Growth by Plasma} Enhanced Chemical Vapor Deposition. Vertically aligned CNTs were grown on the patterned metal catalyst (in this case, $\mathrm{Ni}$ ) by PECVD. The PECVD instrument had a DC plasma chamber consisting of a pair of electrodes in which one electrode was grounded and the other was connected directly to a power supply. The negative DC bias voltage applied to the cathode dissociates the feedstock hydrocarbon gas and generates many carbon-bearing radicals for CNT growth.

Figure 2 shows a schematic diagram of PECVD and the generated plasma (inset). The CNT growth equipment had the following components: a rotary pump to obtain a pressure of $10^{-2}$ Torr, turbo-molecular pump to obtain a pressure of $10^{-6}$ Torr, vacuum chamber to withstand high temperature, mass flow controller (MFC) to measure the flow of acetylene and ammonia gases, vacuum gauge to sense the pressure,
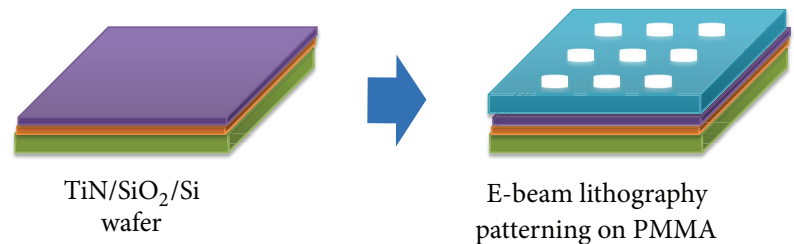

E-beam lithography patterning on PMMA

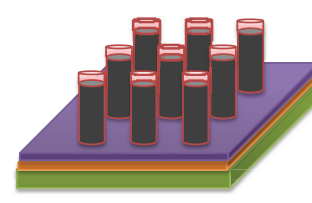

Individual CNTs growth by PECVD

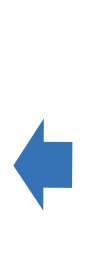

Nickel deposition and lift off
FIGURE 1: Schematic diagram showing microfabrication processes for nanodot arrays and carbon nanotube growth by plasma enhanced chemical vapor deposition (PECVD).

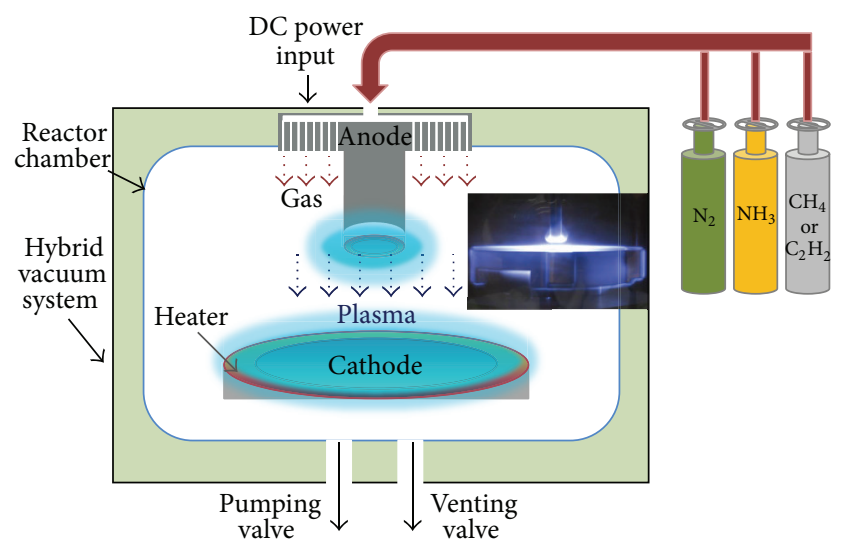

FIGURE 2: Schematic diagram of plasma enhanced chemical vapor deposition and generated plasma (inset).

plasma power supply to ignite the plasma, ceramic heater to obtain temperatures up to $800^{\circ} \mathrm{C}$, heater controller to vary the temperature, cathode chuck on which a wafer piece or whole wafer is placed, and tube fittings for gases and water flow. At the bottom of the cathode chuck, two thermocouples were mounted to measure the temperature of the substrate, which was controlled by the heater controller. Plasma was formed between anode and cathode by applying a DC voltage.

A patterned sample was loaded in the PECVD chamber evacuated by rotary and turbo-molecular pumps to a base pressure $\left(<10^{-6}\right.$ Torr $)$, which was needed for eliminating the impurities and water vapor. When the pressure reached the desired value, the temperature of the heater beneath the cathode was gradually increased to $580^{\circ} \mathrm{C}$. When the temperature became $580^{\circ} \mathrm{C}$, ammonia $\left(\mathrm{NH}_{3}\right)$ gas was introduced into the chamber for 5 15 min without igniting the plasma to etch the catalyst. After subjecting the catalyst to preetching without plasma, acetylene $\left(\mathrm{C}_{2} \mathrm{H}_{2}\right)$ was injected into the chamber and the plasma was ignited to initiate CNT growth. After the CNT growth time, the plasma was turned off and the heater was 
cooled down slowly to prevent CNT damage from a sudden change in temperature. During the CNT growth process, amorphous carbon was deposited on the cathode and heater, which may have caused fluctuations in the plasma. To form stable plasma, the heater and cathode were cleaned after several experiments. Figure 3(a) shows the FESEM images of catalytic nanodot arrays and Figure 3(b) shows the vertically aligned individual carbon nanotube arrays on top of the catalytic nanodot arrays.

\section{Results and Discussion}

3.1. Edge Bead Effect of E-Beam Evaporation. Nanodots were fabricated by e-beam lithography and e-beam evaporation as a catalyst for vertically aligned carbon nanotube growth. In the e-beam process, the edge bead effect generally appears with very small patterns. The edge bead effect occurs when evaporated material is preferentially deposited toward the edge of a pattern compared to the center. Figure 4(a) shows a field emission scanning electron microscopy (FESEM, Zeiss Supra-40 and FEI/Philips XL30 FEG ESEM) image of a $\mathrm{Ni}$ nanodot with a diameter of $400 \mathrm{~nm}$ and a thickness of $5 \mathrm{~nm}$ obtained by e-beam lithography and e-beam evaporation. Because the surface roughness is not easily observed in the FESEM image, the nanodot was scanned by AFM (Figure 4(b)). The figure shows that the catalytic metal was deposited uniformly on the top of the nanodots. Because the deposited layer was thin, no edge bead effect was observed on the nanodot. However, the phenomenon observed for the thick-layered nanodots was different. Figure 4(c) shows an FESEM image of a nanodot with a diameter of $400 \mathrm{~nm}$ and a thickness of $50 \mathrm{~nm}$. From the image, a difference in height was observed at the edge of the nanodot. The AFM scan is shown in Figure 4(d). An unexpected profile with a peak of approximately $5 \mathrm{~nm}$ was observed at the edge of the nanodot (indicated by the white circle). This profile was caused by the edge bead effect during e-beam evaporation. This $\sim 5 \mathrm{~nm}$ peak was obtained in the thicker catalytic layered nanodots and increased the travel distances for carbon atoms in order to reach the interface between the catalytic layer and diffusion barrier in the carbon diffusion process to grow CNTs.

3.2. Brownian Diffusion Distance of an Atom of Particle. A carbon atom of particle is diffused onto or into a metal layer. These atoms of particles are diffused according to the Brownian diffusion distance [20]. The root-mean-square net displacement $\left(x_{\text {rms }}\right)$ along any axis during a time $(t)$ is

$$
x_{\mathrm{rms}}=\sqrt{2 D t} \text {, }
$$

where $D=$ diffusion coefficient $\left[\mathrm{cm}^{2} / \mathrm{s}\right], t=$ time $[\mathrm{s}]$. From this formula, the thicker a layer, the longer diffusion time of an atom of particle.

3.3. Carbon Atom Diffusion and Lift-Off Catalyst. CNTs can be grown by following a three-step procedure as follows: (1) decomposition of the hydrocarbon gas (in this study, acetylene $\left(\mathrm{C}_{2} \mathrm{H}_{2}\right)$ ) over a catalytic metal $(\mathrm{Ni}),(2)$ diffusion of the carbon atoms through the bulk and surface of the catalyst, and (3) subsequent precipitation of the carbon atoms beneath the catalyst $[17,19]$. In the second step, carbon atoms must reach the interface of the catalytic layer and the diffusion barrier. Figure 5(a) shows the 3D AFM image of the catalytic nanodot with a thickness of $50 \mathrm{~nm}$ and a diameter of $400 \mathrm{~nm}$. As mentioned above, the unexpected over deposition at the edge of the nanodot was clearly observed as shown in Figure 5(a) (the white circle). This unexpected peak made the catalytic layer thicker at the edge of the nanodot compared with its center. Figure 5(b) shows a schematic diagram of a carbon atom's diffusion in the catalytic nanodot. As shown in Figure 5(b), carbon atoms diffuse to the interface between the catalytic layer and the diffusion barrier through a bulk or surface diffusion process. Carbon atoms at the center of the nanodots could diffuse faster than those at the edge of the dot because of the thicker catalytic layer at the edge of the nanodot. Carbon atoms accumulated earlier in the center of the nanodot caused a specific catalytic area to lift off, as shown in Figure 5(b). Consequently, the resulting carbon nanotube has a smaller diameter than expected with the thick catalytic nanodots.

3.4. Vertically Aligned Individual Carbon Nanotubes and Relation between Carbon Nanotube Diameter and Catalyst Thickness. The CNT's electric dipole, accruing from the electric field exerted on the CNT, interacts with the DC electric field and experiences an alignment torque, developing stresses at the interface between the CNT and the catalyst particle. Because the diffusion flux depends on the stress gradient as well as the concentration gradient, stressinduced fluctuations in the diffusion rate can be primary mechanism for the vertical alignment of the CNTs. Figures 6(a), 6(b), and 6(c) show the vertically aligned individual carbon nanotubes made using catalyst thicknesses of 5, 30, and $50 \mathrm{~nm}$, respectively. The individual carbon nanotube from a $5 \mathrm{~nm}$ thick catalyst had the same diameter as that of the initial nanodot, that is, a diameter of $400 \mathrm{~nm}$. In the case of $30 \mathrm{~nm}$ and $50 \mathrm{~nm}$ catalyst thicknesses, individual carbon nanotubes with small diameters were synthesized. For the 30 $\mathrm{nm}$ thick catalytic nanodot, the resulting carbon nanotube had a $30 \%$ smaller diameter. Furthermore, the diameter of the individual carbon nanotube was reduced by $40 \%$ when $50 \mathrm{~nm}$ thick catalytic nanodots were used. Figure 6(d) shows the relation between the thickness of the catalyst layer and the diameter of the individual carbon nanotubes. From this work, we found that the thickness control of catalytic nanodots is a very important parameter for obtaining vertically aligned individual carbon nanotubes with predictable diameter.

\section{Conclusions}

In this work, the effect of catalyst layer thickness on the diameter of vertically aligned individual carbon nanotubes produced by PECVD was investigated. For the growth of individual carbon nanotubes, catalytic nanodot arrays were fabricated by e-beam lithography and e-beam evaporation. During e-beam evaporation, the edge effect was observed in 


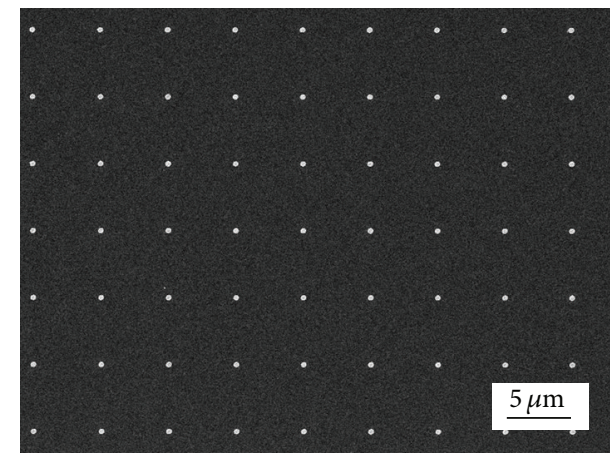

(a)

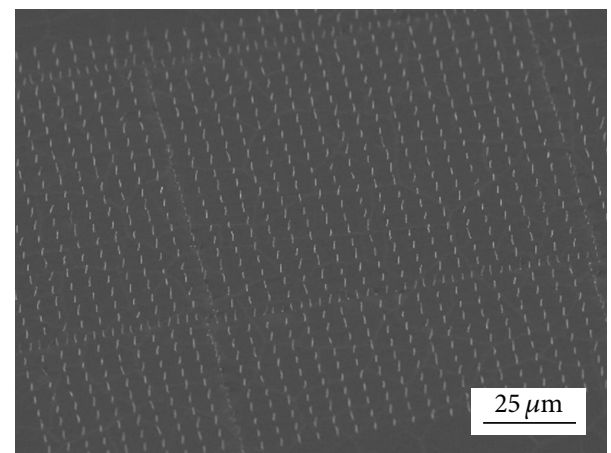

(b)

FIGURE 3: Field emission scanning probe microscopy (FESEM) images of (a) catalytic nanodot array and (b) vertically aligned individual carbon nanotube array.

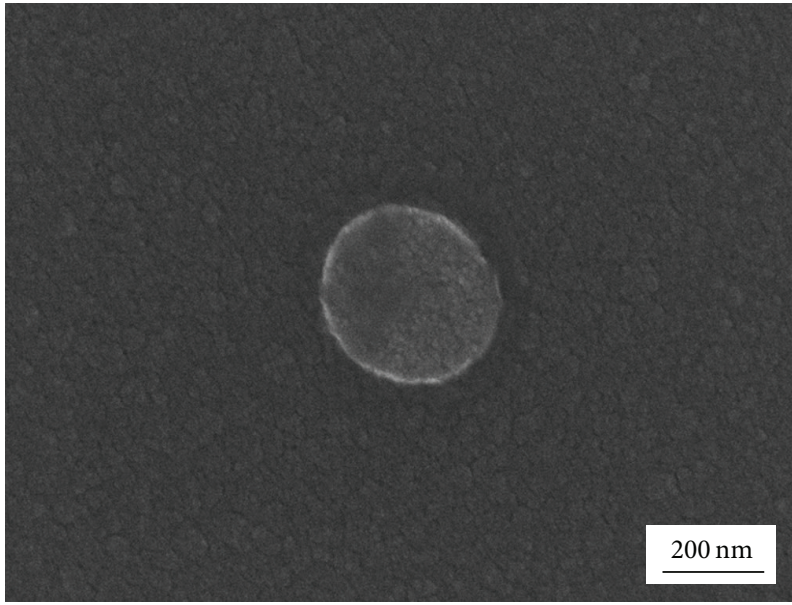

(a)

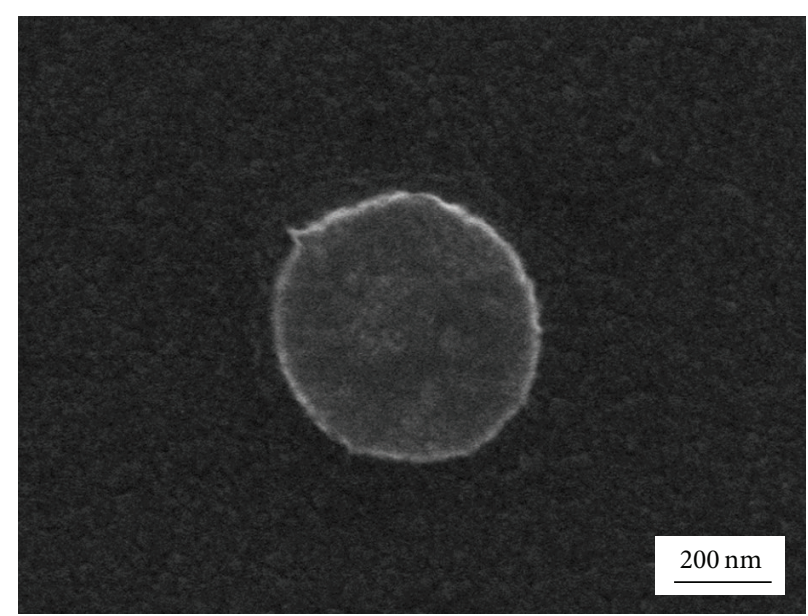

(c)

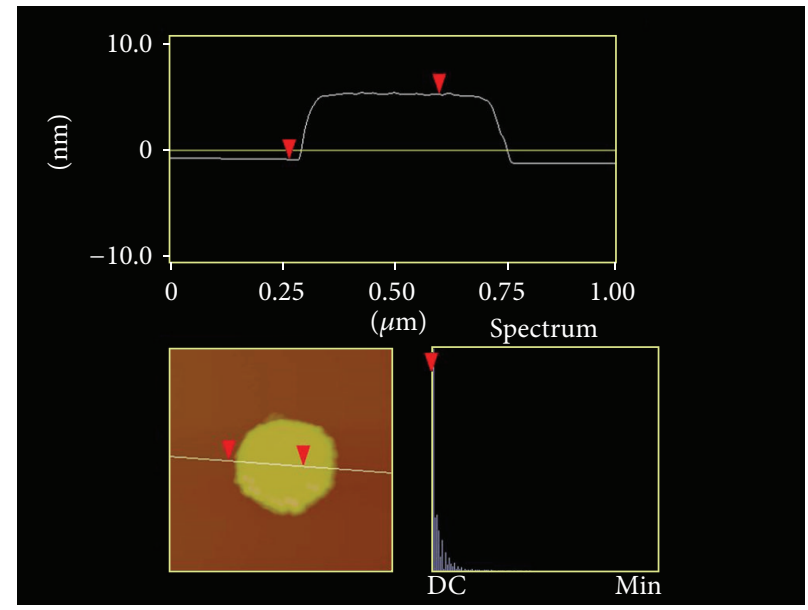

(b)

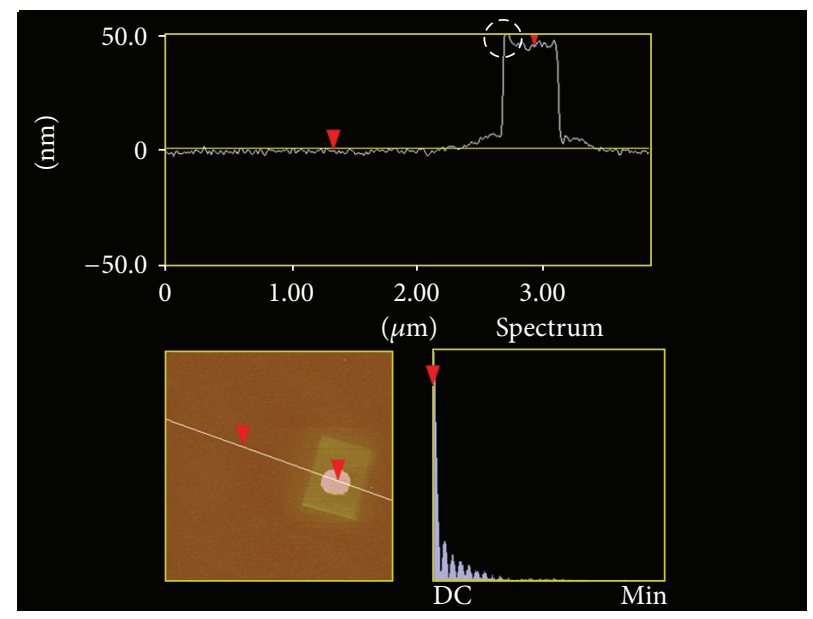

(d)

FIGURE 4: (a) Scanning electron microscopy (SEM) image and (b) atomic force microscopy (AFM) scanning profile of catalytic nanodot with a thickness of $5 \mathrm{~nm}$; (c) SEM image and (d) AFM scanning profile of catalytic nanodot with a thickness of $50 \mathrm{~nm}$. 


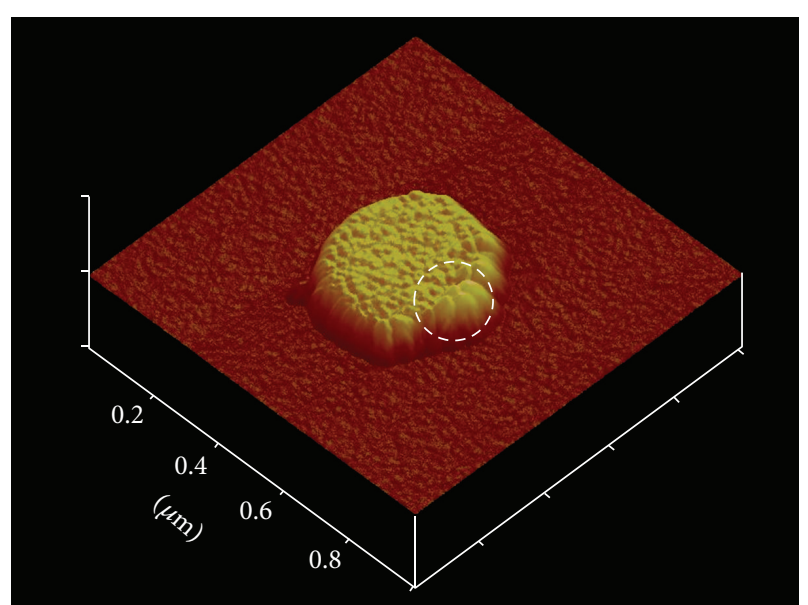

(a)

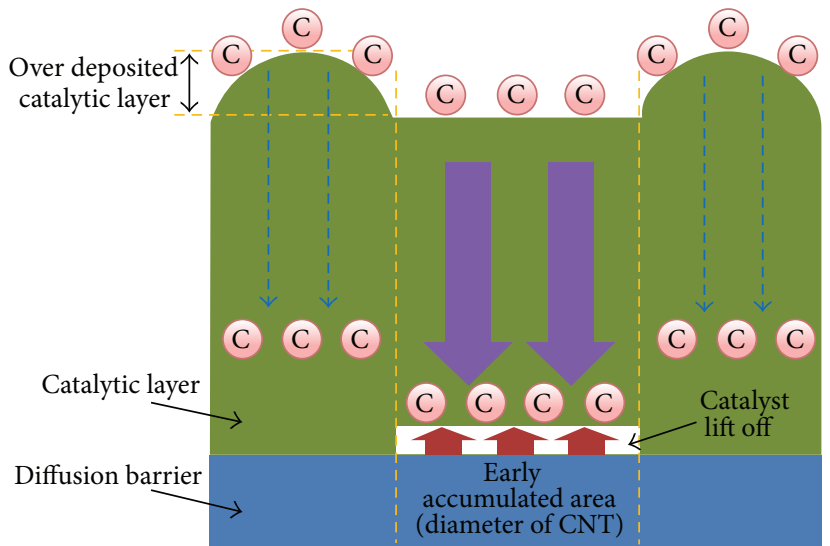

(b)

FIgURE 5: (a) Three-dimensional (3D) atomic force microscopy (AFM) scanning image of $50 \mathrm{~nm}$ thick nanodot and (b) schematic diagram of carbon atoms' diffusion.

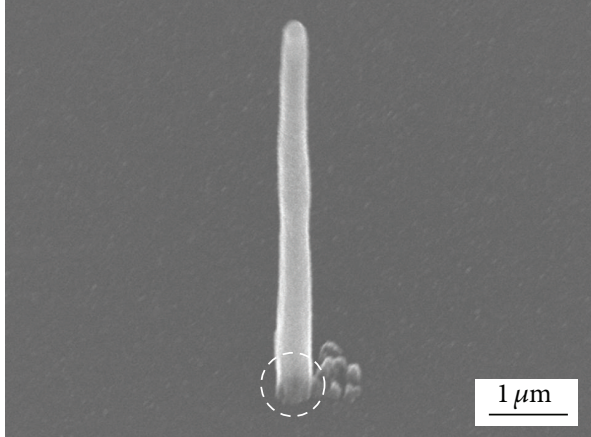

(a)

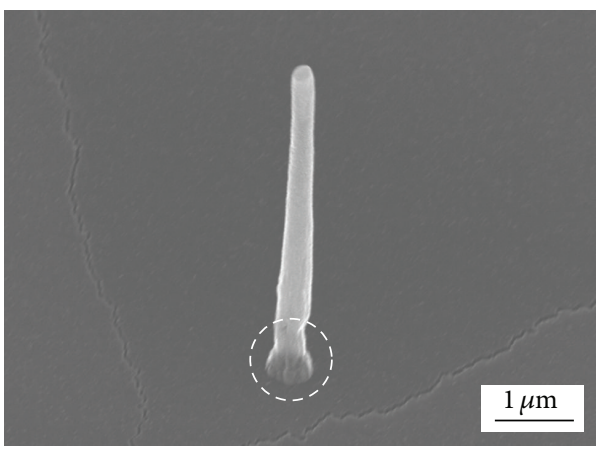

(c)

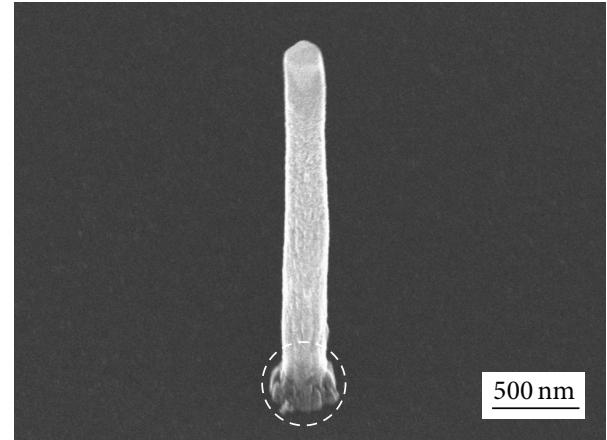

(b)

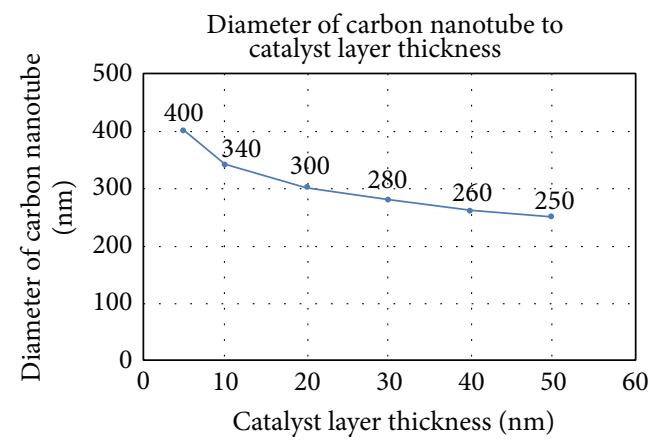

(d)

FIGURE 6: Scanning electron microscopy (SEM) images of vertically aligned carbon nanotubes from catalytic nanodots with thicknesses of (a) $5 \mathrm{~nm}$, (b) $30 \mathrm{~nm}$, and (c) $50 \mathrm{~nm}$; (d) relation between thickness of catalytic nanodots and diameter of carbon nanotubes.

catalytic nanodots, because the diameters of the dots were very small. Because of the edge effect, the catalytic metal was deposited preferentially toward the edge of the dot resulting in a larger thickness than desired. When growing vertically aligned individual carbon nanotubes by PECVD, thick catalytic nanodots reduced the diameter of the synthesized carbon nanotubes. The reduction occurred because carbon atoms diffused faster near the center of dots than at the edges. The carbon atoms diffused to the interface between the catalytic nanodot and the diffusion barrier and accumulated near the center, causing the center of the catalytic nanodot to lift off faster than the edge of the dot.

From the experiments, individual carbon nanotubes with the same diameter as that of the catalytic nanodots $(400 \mathrm{~nm})$ were obtained when the catalyst thickness was $5 \mathrm{~nm}$. For $50 \mathrm{~nm}$ thick catalytic nanodots, a carbon nanotube with diameter of $260 \mathrm{~nm}$ (reduction by $\sim 40 \%$ ) was obtained. We found that the thicker the catalytic layer, the greater the 
reduction in the diameter of the individual carbon nanotubes. From the FESEM and AFM images, we verified the relation between the diameter of the carbon nanotubes and the catalytic layer thickness of the nanodots.

Diameter control of vertically aligned individual carbon nanotubes is very important and useful for the application of CNTs in electric and energy-based devices and for scanning bio- and nanomaterials. Furthermore, the diametercontrolled carbon nanotubes could be applied to a contrast medium for magnetic resonance imaging.

\section{Conflict of Interests}

The authors declare that there is no conflict of interests regarding the publication of this paper.

\section{Acknowledgment}

This work was supported by the 2012 Inje University research Grant.

\section{References}

[1] S. Iijima, "Helical microtubules of graphitic carbon," Nature, vol. 354, no. 6348, pp. 56-58, 1991.

[2] T. Rueckes, K. Kim, E. Joselevich, G. Y. Tseng, C. Cheung, and C. M. Lieber, "Carbon nanotube-based nonvolatile random access memory for molecular computing," Science, vol. 289, no. 5476, pp. 94-97, 2000.

[3] J. Kong, N. R. Franklin, C. Zhou et al., "Nanotube molecular wires as chemical sensors," Science, vol. 287, no. 5453, pp. 622$625,2000$.

[4] K. Besteman, J. Lee, F. G. M. Wiertz, H. A. Heering, and C. Dekker, "Enzyme-coated carbon nanotubes as single-molecule biosensors," Nano Letters, vol. 3, no. 6, pp. 727-730, 2003.

[5] H. W. Lee, S. H. Kim, C. S. Han, and Y. K. Kwak, "Nanoscale fabrication of a single multiwalled carbon nanotube attached atomic force microscope tip using an electric field," Review of Scientific Instruments, vol. 76, no. 4, Article ID 046108, 2005.

[6] H. W. Lee, S. H. Kim, Y. K. Kwak, E. S. Lee, and C. S. Han, “The effect of the shape of a tip's apex on the fabrication of an AFM tip with an attached single carbon nanotube," Sensors and Actuators A, vol. 125, no. 1, pp. 41-49, 2005.

[7] T. A. El-Aguizy, J.-H. Jeong, Y. B. Jeon, W. Z. Li, Z. F. Ren, and S. G. Kim, “Transplanting carbon nanotubes," Applied Physics Letters, vol. 85, no. 24, pp. 5995-5997, 2004.

[8] Z. F. Ren, Z. P. Huang, J. W. Xu et al., "Synthesis of large area of well-aligned carbon nanotubes on glass," Science, vol. 282, no. 5391, pp. 1105-1107, 1998.

[9] S. Kim, H. W. Lee, and S. G. Kim, "Transplanting assembly of carbon-nanotube-tipped atomic force microscope probes," Applied Physics Letters, vol. 94, no. 19, Article ID 193102, 2009.

[10] M. Chhowalla, K. B. K. Teo, C. Ducati et al., "Growth process conditions of vertically aligned carbon nanotubes using plasma enhanced chemical vapor deposition," Journal of Applied Physics, vol. 90, no. 10, pp. 5308-5317, 2001.

[11] A. V. Melechko, V. I. Merkulov, T. E. Mcknight et al., "Vertically aligned carbon nanofibers and related structures: controlled synthesis and directed assembly," Journal of Applied Physics, vol. 97, no. 4, Article ID 041301, 2005.
[12] M. Meyyappan, L. Delzeit, A. Cassell, and D. Hash, "Carbon nanotube growth by PECVD: a review," Plasma Sources Science and Technology, vol. 12, no. 2, pp. 205-216, 2003.

[13] Y. Tu, Z. P. Huang, D. Z. Wang, J. G. Wen, and Z. F. Ren, "Growth of aligned carbon nanotubes with controlled site density," Applied Physics Letters, vol. 80, no. 21, pp. 4018-4020, 2002.

[14] Y. Wang, J. Rybczynski, D. Z. Wang et al., "Periodicity and alignment of large-scale carbon nanotubes arrays," Applied Physics Letters, vol. 85, no. 20, pp. 4741-4743, 2004.

[15] V. I. Merkulov, D. K. Hensley, A. V. Melechko, M. A. Guillorn, D. H. Lowndes, and M. L. Simpson, "Control mechanisms for the growth of isolated vertically aligned carbon nanofibers," Journal of Physical Chemistry B, vol. 106, no. 41, pp. 10570-10577, 2002.

[16] A. V. Melechko, T. E. Mcknight, D. K. Hensley et al., "Large-scale synthesis of arrays of high-aspect-ratio rigid vertically aligned carbon nanofibres," Nanotechnology, vol. 14, no. 9, pp. 10291035, 2003.

[17] S. Hofmann, M. Cantoro, B. Kleinsorge et al., "Effects of catalyst film thickness on plasma-enhanced carbon nanotube growth," Journal of Applied Physics, vol. 98, no. 3, Article ID 034308, 2005.

[18] V. I. Merkulov, D. H. Lowndes, Y. Y. Wei, G. Eres, and E. Voelkl, "Patterned growth of individual and multiple vertically aligned carbon nanofibers," Applied Physics Letters, vol. 76, no. 24, pp. 3555-3557, 2000.

[19] K. B. K. Teo, S. B. Lee, M. Chhowalla et al., "Plasma enhanced chemical vapour deposition carbon nanotubes/nanofibreshow uniform do they grow?" Nanotechnology, vol. 14, no. 2, pp. 204-211, 2003.

[20] W. C. Hinds, Aerosol Technology: Properties, Behavior, and Measurement of Airborne Particles, John Wiley \& Sons, 1999. 

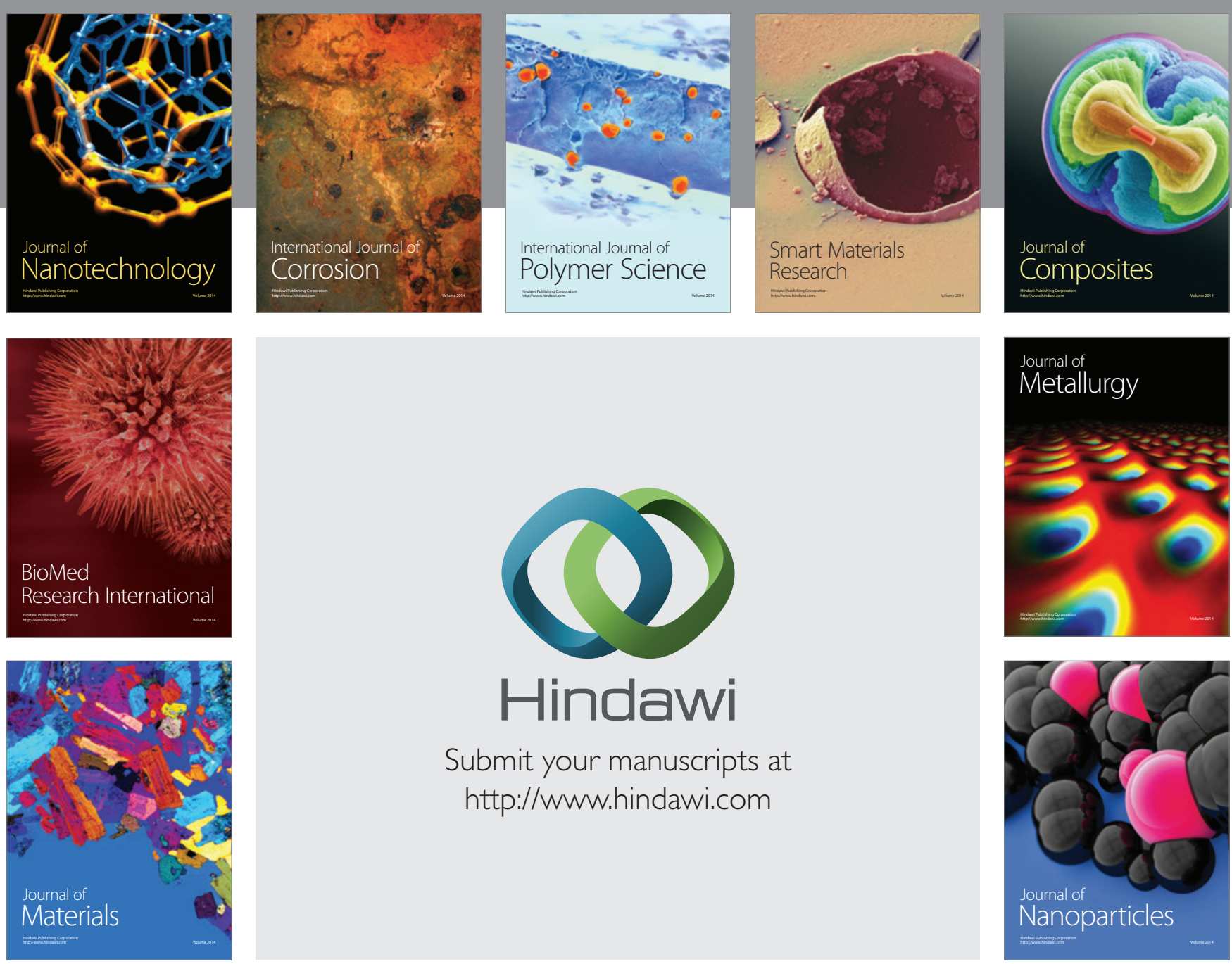

Submit your manuscripts at http://www.hindawi.com
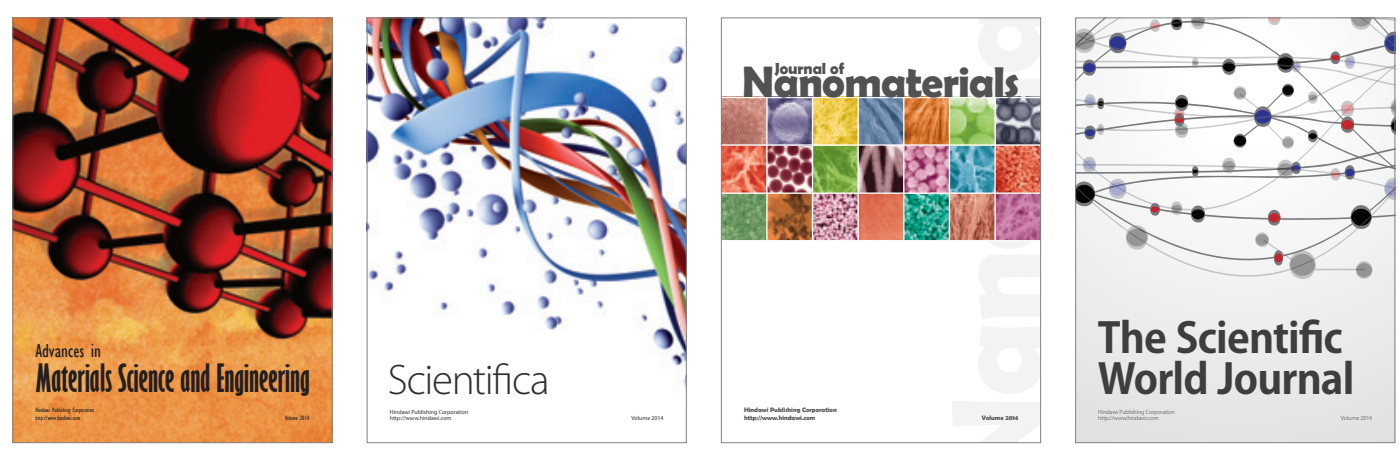

\section{The Scientific World Journal}
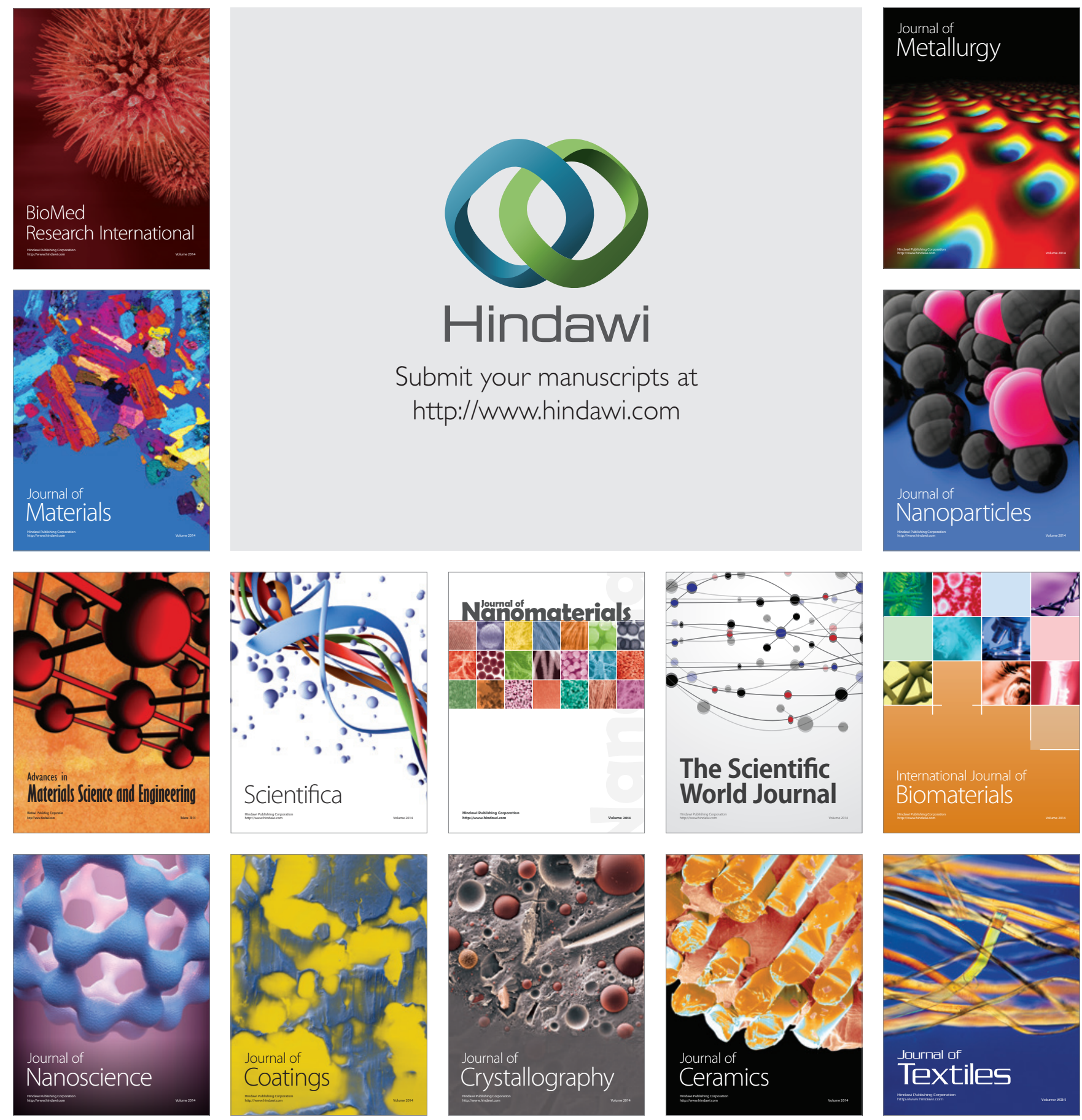